\title{
Solubility of 2-Hydroxybenzoic Acid in Select Organic Solvents at 298.15 K
}

\author{
Karina M. De Fina, Tina L. Sharp, Lindsay E. Roy, and William E. Acree J r.*
}

Department of Chemistry, University of North Texas, Denton, Texas 76203-5070

\begin{abstract}
Experimental solubilities are reported for 2-hydroxybenzoic acid dissolved in 1-propanol, 2-propanol, 1-butanol, 2-butanol, 2-methyl-1-propanol, 2-methyl-2-propanol, 1-pentanol, 1-octanol, dibutyl ether, 1,4dioxane, tetrahydrofuran, ethyl acetate, butyl acetate, 2-propanone, 2-butanone, and cyclohexanone at $298.15 \mathrm{~K}$. Results of these measurements reveal that the observed solubilities in the eight al cohol solvents fall within a fairly narrow mole fraction range of each other. 2-Hydroxybenzoic acid is more soluble in both 1,4-dioxane and tetrahydrofuran than in any of the other al cohol, ester, or ketone solvents studied.
\end{abstract}

\section{Introduction}

Partition coefficients describing the distribution of a solute between two completely immiscible and/or partly miscible gas/liquid and liquid/liquid phases are often needed in chemical engineering computations involving chromatographic separations and selection of suitable organic solvents for extracting organic compounds from aqueous solutions. For many organic solutes, experimentally determined 1-octanol/water partition coefficients are readily available in the published chemical, pharmaceuti$\mathrm{cal}$, and engineering literature. Measured partition coefficient data for other immiscible liquid/liquid systems are more scarce. To address this need, researchers have developed predictive methods for generating desired quantities.

Of the predictive expressions that have been suggested in recent years, linear solvation energy relationships (LSERs) offer perhaps the most general method in that the derived correlations enable not only estimation of partition coefficients but also estimation of other physicochemical and biochemical properties as well. Proposed LSERs have taken several different mathematical forms, depending upon whether one wishes to predict gas/organic solvent partition coefficients (as log L) (Abraham et al., 1990)

$$
\begin{gathered}
\log L=c+r \cdot R_{2}+s \cdot \pi_{2}{ }^{H}+a \cdot \sum \alpha_{2}{ }^{H}+b \cdot \sum \beta_{2}{ }^{H}+ \\
I \cdot \log L^{16} \\
\log L=c+d \cdot \delta_{2}+s \cdot \pi_{2}{ }^{H}+a \cdot \sum \alpha_{2}{ }^{H}+b \cdot \sum \beta_{2}{ }^{H}+ \\
I \cdot \log { }^{16} \\
\log L=c+r \cdot R_{2}+q \cdot \mu_{2}{ }^{2}+a \cdot \sum \alpha_{2}{ }^{H}+b \cdot \sum \beta_{2}{ }^{H}+ \\
I \cdot \log L^{16}
\end{gathered}
$$

or water/organic solvent partition coefficients (as log P) (Abraham et al., 1990; Meyer and Maurer, 1993 and 1995)

$$
\log \mathrm{P}=\mathrm{c}+\mathrm{r} \cdot \mathrm{R}_{2}+\mathrm{s} \cdot \pi_{2}{ }^{\mathrm{H}}+\mathrm{a} \cdot \sum \alpha_{2}{ }^{\mathrm{H}}+\mathrm{b} \cdot \sum \beta_{2}{ }^{\mathrm{H}}+\mathrm{v} \cdot \mathrm{V}_{\mathrm{x}}
$$

* To whom correspondence should be addressed. E-mail: acree@ unt.edu. Fax: (940) 565-4318.

$$
\log \mathrm{P}=\mathrm{c}+\mathrm{d} \cdot \delta_{2}+\mathrm{s} \cdot \pi_{2}+\mathrm{a} \cdot \alpha_{2}+\mathrm{b} \cdot \beta_{2}+\mathrm{v} \cdot \mathrm{V}_{\mathrm{x}}
$$

where $R_{2}$ and $V_{x}$ refer to the excess molar refraction and the McGowan volume of the solute, respectively, $\pi_{2}{ }^{H}$ (and $\pi_{2}$ ) is the solute dipolarity/polarizability descriptor, and $\sum \alpha_{2}{ }^{H}$ (and $\alpha_{2}$ ) and $\sum \beta_{2}{ }^{\mathrm{H}}$ (and $\beta_{2}$ ) are measures of the solute's hydrogen-bond acidity and hydrogen-bond basicity, respectively. $\delta_{2}$ is a polarizability parameter which was preset by Kamlet et al. (1988) to 0.0 in the case of nonpolychlorinated al iphatic sol utes, to 0.5 for polychl orinated aliphatic solutes, and to 1.0 for aromatic solutes. Numerical values of the solute descriptors are available in several published tabulations for $500+$ common organic molecules and inorganic gas solutes (Abraham 1993a,b; Kamlet et al., 1988; Meyer and Maurer, 1995). For any given solvent, numerical values of the various curve-fit coefficients (c, r, s, a, b, d, l, $q$, and $v$ ) are deduced by regressing experimental partition coefficient data in accordance with eqs 1-5. At the present time coefficients are available for only 50 or so different water/organic solvent and gas/solvent systems (Meyer and Maurer, 1993 and 1995; Abraham et al., 1998).

We, in collaboration with the Abraham research group, are in the process of devel oping correlational equations for additional solvent systems and new methodologies for calculating solute descriptors. 1-Propanol was the first solvent for which we (Abraham et. al., 1999) reported LSER solvent coefficients. It should be noted that 1-propanol is completely miscible with water at $298.15 \mathrm{~K}$, and the $\log \mathrm{P}$ and $\log L$ values used in developing the correlation equations were deduced from measured infinite dilution activity and sol ubility data for sol utes dissolved in neat 1-propanol.

Continued development of LSERs for describing the partitioning behavior of solutes requires that a large database be available for each of the different systems that is to be studied. For this reason, 2-hydroxybenzoic acid solubilities were measured in 16 organic solvents. Results of these measurements, combined with our previously published solubility data for polycyclic aromatic hydrocarbon solutes, will be used in subsequent studies to develop gas/liquid partition and water/organic solvent partition correlations based upon eqs $1-5$.

\section{Experimental Methods}

2-Hydroxybenzoic acid (Aldrich 99+\%, ACS Reagent Grade) was dried in an oven for several hours at $80^{\circ} \mathrm{C}$ and 
Table 1. Experimental Mole Fraction Solubilities $x_{A}^{\text {sat }}$ of 2-Hydroxybenzoic Acid, 4-Hydroxybenzoic Acid, and Benzoic Acid in Select Organic Solvents at 298.15 K

\begin{tabular}{lccc}
\hline \multicolumn{1}{c}{ solvent } & & mole fraction solubility & \\
\cline { 2 - 4 } 1-propanol & 2- $\mathrm{HOC}_{6} \mathrm{H}_{4} \mathrm{COOH}$ & $4-\mathrm{HOC}_{6} \mathrm{H}_{4} \mathrm{COOH}$ & $\mathrm{C}_{6} \mathrm{H}_{5} \mathrm{COOH}$ \\
2-propanol & 0.1636 & $0.1084^{\mathrm{a}}$ & $0.1791^{\mathrm{b}}$ \\
1-butanol & 0.1789 & $0.1743^{\mathrm{c}}$ & $0.1937^{\mathrm{b}}$ \\
2-butanol & 0.1646 & $0.1154^{\mathrm{a}}$ & $0.2016^{\mathrm{b}}$ \\
2-methyl-1-propanol & 0.1869 & $0.0901^{\mathrm{a}}$ & $0.1524^{\mathrm{b}}$ \\
2-methyl-2-propanol & 0.1430 & & $0.1832^{\mathrm{b}}$ \\
1-pentanol & 0.2193 & $0.1145^{\mathrm{a}}$ & $0.1987^{\mathrm{b}}$ \\
1-octanol & 0.1611 & $0.1032^{\mathrm{a}}$ & $0.2853^{\mathrm{b}}$ \\
dibutyl ether & 0.2143 & & $0.3348^{\mathrm{c}}$ \\
1,4-dioxane & 0.09185 & & $0.1649^{\mathrm{b}}$ \\
tetrahydrofuran & 0.2945 & $0.1637^{\mathrm{c}}$ \\
ethyl acetate & 0.3642 & & $0.1699^{\mathrm{b}}$ \\
butyl acetate & 0.1425 & & \\
2-propanone & 0.1363 & & \\
2-butanone & 0.1817 & & \\
cyclohexanone & 0.1852 & & \\
\end{tabular}

${ }^{a}$ Experimental data taken from Martin et al. (1984). ${ }^{b}$ Experimental data taken from Beerbower et al. (1984). ${ }^{c}$ Experimental data taken from Lin and Nash (1993).

used without further purification. The purity of 2-hydroxybenzoic acid was $99.86 \pm 0.12$ mass $\%$, as determined by five volumetric titrations using a freshly standardized sodium hydroxide titrant and a phenolphthalein indicator. 1-Propanol (Aldrich, 99+\%, anhydrous), 2-propanol (Aldrich, 99+\%, anhydrous), 1-butanol (Aldrich, HPLC, 99.8+\%), 2-butanol (Aldrich, 99+\%, anhydrous), 2-methyl-1-propanol (Aldrich, 99.5\%, anhydrous), 2-methyl-2-propanol (Arco Chemical Company, 99+\%), 1-pentanol (Aldrich, 99+\%), 1-octanol (Aldrich, 99+\%, anhydrous), dibutyl ether (Aldrich, 99\%), 1,4-dioxane (Aldrich, 99.8\%, anhydrous), tetrahydrofuran (Aldrich, 99.9\%, anhydrous), ethyl acetate (Aldrich, HPLC, 99.9\%), butyl acetate (Aldrich HPLC, 99.7\%), 2-propanone (Aldrich HPLC, 99.9+\%), 2-butanone (Aldrich HPLC, 99.5+\%), and cyclohexanone (Aldrich, 99.8\%) were stored over molecular sieves before use. Gas chromatographic analysis showed solvent purities to be $99.7 \mathrm{~mol} \%$ percent or better.

Excess solute and solvent were placed in amber glass bottles and allowed to equilibrate in a constant-temperature water bath at $25.0 \pm 0.1{ }^{\circ} \mathrm{C}$ for at least 3 days (often longer). Attainment of equilibrium was verified both by repetitive measurements after several additional days and by approaching equilibrium from supersaturation by preequilibrating the solutions at a higher temperature. Aliquots of saturated 2-hydroxybenzoic acid solutions were transferred through a coarse filter into a tared volumetric flask to determine the amount of sample and diluted quantitatively with methanol for spectrophotometric analysis at $304 \mathrm{~nm}$ on a Bausch and Lomb Spectronic 2000. Concentrations of the dilute solutions were determined from a Beer-Lambert law absorbance versus concentration working curve for nine standard solutions. The concentration of 2-hydroxybenzoic acid in the standard solutions varied from $8.83 \times 10^{-5} \mathrm{M}$ to $4.42 \times 10^{-4} \mathrm{M}$. The calculated molar absorptivity of $\epsilon \approx 3985 \mathrm{~L} \mathrm{~mol}^{-1} \mathrm{~cm}^{-1}$ was constant over this concentration range.

Experimental molar concentrations were converted to (mass/mass) solubility fractions by multiplying by the molar mass of 2-hydroxybenzoic acid, the volume(s) of the vol umetric flask(s) used, and any dilutions required to place the measured absorbances on the Beer-Lambert law absorbance versus concentration working curve and then dividing by the mass of the saturated solution analyzed.
Mole fraction solubilities were computed from (mass/mass) solubility fractions using the molar masses of the solute and solvent. 2-Propanone, 2-butanone, and cyclohexanone do absorb at the analysis wavelength. 2-Hydroxybenzoic acid was sufficiently soluble in these three solvents that by the time the saturated solutions were appropriately diluted for the measured absorbances to fall on the BeerLambert Law curve, the solvent background absorbances were negligible.

\section{Results and Discussion}

Experimental 2-hydroxybenzoic acid solubilities $x_{A}^{\text {sat }}$, in 16 organic solvents studied are listed in Table 1. Numerical values represent the average of between four and eight independent determinations, with the measurements being reproducible to $\pm 1.8 \%$. We were unable to find any published solubility data in the chemical literature against which to compare directly our measured values. We did find experimental solubility data for benzoic acid (Beerbower et al., 1984; Lin and Nash, 1993) and 4-hydroxybenzoic acid (Martin et al., 1984), and these values have been tabulated in the third and fourth columns of Table 1. All three solutes are expected to have different solubilities in any given solvent because of differences in their respective melting point temperatures and enthalpies of fusion. The three crystalline solutes are benzoic acid derivatives, however, and to a first approximation one would expect that the molecular interactions between 2-hydroxybenzoic acid and a given solvent would be similar to the molecular interactions between benzoic acid and the specified organic solvent. These interactions, in turn, should be similar to those between 4-hydroxybenzoic acid and the organic solvent under consideration. Naturally, there might be some slight differences/variations caused by the location of the hydroxyl functional group.

Examination of Table 1 reveals that, in the case of benzoic acid, the published mole fraction solubilities are fairly close together for the alcohol solvents. Benzoic acid is more soluble in 2-propanol than in 1-propanol, and more soluble in 1-butanol than in 2-butanol. Many of the same trends are observed for both 2-hydroxybenzoic acid and 4-hydroxybenzoic acid. Both hydroxybenzoic acids are more soluble in 2-propanol than 1-propanol. A reversal in 
1264 J ournal of Chemical and Engineering Data, Vol. 44, No. 6, 1999

solubility order is noted in the two butanol solvents. Measured solubilities in the alcohol solvents do fall within a narrow mole fraction range, particularly if 2-methyl-1propanol is excluded. Benzoic acid and 2-hydroxybenzoic acid are more soluble in 1,4-dioxane and tetrahydrofuran than in any of the alcohol, ketone, and ester solvents studied.

\section{Literature Cited}

Abraham, M. H. Scales of Solute Hydrogen-Bonding: Their Construction and Application to Physicochemical and Biochemical Processes. Chem. Soc. Rev. 1993a, 22, 73-83.

Abraham, M. H. Hydrogen Bonding. 31. Construction of a Scale of Solute Effective or Summation Hydrogen-Bond Basicity. J . Phys. Org. Chem. 1993b, 6, 660-684.

Abraham, M. H.; Whiting, G. S.; Doherty, R. M.; Shuely, W. J. Hydrogen Bonding. XV. A New Characterisation of the McReynolds 77-Stationary Phase Set. J. Chromatogr. 1990, 518, 329-348.

Abraham, M. H.; Green, C. E.; Acree, W. E., J r.; Hernández, C. E.; Roy, L. E. Descriptors for Solutes from the Solubility of Solids: trans-Stilbene as an Example. J . Chem. Soc., Perkin Trans. 2 1998, 2677-2681.

Abraham, M. H.; Le, J .; Acree, W. E., J r.; Carr, P. W. The Solubility of Gases and Vapours in Propan-1-ol at 298 K. J . Phys. Org. Chem. 1999, in press.

Beerbower, A.; Wu, P. L.; Martin, A. Expanded Solubility Parameter Approach I: Naphthal ene and Benzoic Acid in Individual Solvents. J . Pharm. Sci. 1984, 73, 179-188.
Kamlet, M. J .; Doherty, R. M.; Abraham, M. H.; Marcus, Y.; Taft, R. W. Linear Solvation Energy Relationships. 46. An Improved Equation for Correlation and Prediction of Octanol/Water Partition Coefficients of Organic N onelectrolytes (Including Strong Hydrogen Bond Donor Solutes). J . Phys. Chem. 1988, 92, 5244-5255.

Lin, H.-M.; Nash, R. A. An Experimental Method for Determining the Solubility Parameter of Organic Nonelectrolytes. J . Pharm. Sci. 1993, 82, 1018-1026.

Martin, A.; Wu, P. L.; Beerbower, A. Expanded Solubility Parameter Approach II: p-Hydroxybenzoic Acid and Methyl p-Hydroxybenzoate in Individual Solvents. J. Pharm. Sci. 1984, 73, 188-194.

Meyer, P.; Maurer, G. Correlation of Partition Coefficients of Organic Solutes Between Water and an Organic Solvent. An Application of the Linear Solvation Energy Relationship. Ind. Eng. Chem. Res. 1993, 32, 2105-2110.

Meyer, P.; Maurer, G. Correlation and Prediction of Partition Coefficients of Organic Solutes Between Water and an Organic Solvent with a Generalized Form of the Linear Solvation Energy Relationship. Ind. Eng. Chem. Res. 1995, 34, 373-381.

\section{Acknowledgment}

Received for review May 17, 1999. Accepted J uly 27, 1999. T.L.S. thanks the U niversity of North Texas and the U.S. Department of Education for support provided under the Ronald E. McNair Postbaccalaureate Achievement Program.

J E990134W 